\title{
DSTATCOM with Artificial Intelligent Controller for Voltage Sag Mitigation
}

\author{
T.Divya Sree ${ }^{1}$, M.Devi Shankar ${ }^{2}$ \\ PG Student, EEE, V.R.Siddhartha College, Vijayawada, India ${ }^{1}$ \\ Associate Professor, EEE, V.R.Siddhartha College, Vijayawada, India ${ }^{2}$
}

\begin{abstract}
Fault at Distribution level cause transient voltage sag in entire system or large part of it. Disturbances such as voltage sag, swell, short duration interruption, transient and harmonic occurs. Fault on $11 \mathrm{Kv}$ system or at load point affects voltage profile of other feeders. Unsymmetrical fault effect on distribution line and voltage restoration using DSTATCOM which is a custom power device. This paper deals with Fuzzy logic controller implemented for the distributed system. The proposed method is implemented by using MATLAB/SIMULINK.
\end{abstract}

Keywords: DSTATCOM, Voltage sag, FIS, MATLAB SIMULINK.

\section{INTRODUCTION}

Power quality is major concern in the present era. Sophisticated devices made power quality important. The custom power device performance is very sensitive to power supply quality. The power quality problem is an occurrence manifested as a nonstandard current, voltage or frequency which in turn results in a failure of end user equipments.

Power quality problems comprises of a wide range of disturbances such as voltage sags, voltage swells, flickers, harmonics, distortion, impulse, transient and interruptions. Among this problem, voltage sags is the frequently occurring problems in terms of power quality problems. In the IEC terminology, IEC 60050- 604, 1998 defines voltage sag as a "sudden reduction of the voltage at a point in the electrical system, followed by voltage recovery after a short period of time, from the half a cycle to a few seconds". Likewise, in more clearly A sag, as defined by IEEE Standard 1159, IEEE Recommended Practice for Monitoring Electric Power Quality, is "a decrease in RMS voltage or current at the power frequency for durations from 0.5 cycles to 1 minute, reported as the remaining voltage". Voltage sags are appearing due to faults, transformer energizing and motor starting. Typical values are in between 0.1p.u. and 0.9p.u. and the typical clearing fault time range from three to thirty cycles depending on the fault current magnitude and the type of over current detection and interruption [1].

There are different ways to mitigate power quality problems in transmission and distribution systems. The sophisticated devices are custom power device for the low voltage distribution, for improving the poor quality and reliability of supply affecting the sensitive loads. Custom power device is very similar to the FACTS. Most widely known as Custom power devices are DSTATCOM, UPQC and DVR. Among these, D-STATCOM is one of the most effective devices. A Distribution Static Compensator (DSTATCOM) is the most efficient and effective modern custom power device used in power distribution networks. Its advantage includes lower cost, smaller size, and its fast dynamic response to the disturbance.
The importance of this paper is to resolve voltage sag problem manifested in voltage deviations that result in failure of customer equipment. The model of the custom power device, namely, D-STATCOM and its control application to mitigate voltage sag[2].The schematic representation of DSTATCOM is represented in figure1.

The D-STATCOM (Distribution Static Compensator) consists of a Voltage Source Converter (VSC), a dc energy storage device, a coupling transformer connected in shunt to the distribution network through the coupling transformer. Suitable for the adjustment of the phase and magnitude of the D-STATCOM output voltages allows effective control of active and reactive power exchanges between the D-STATCOM and the ac system. Such configuration allows the device to absorb or generate controllable active and reactive power.

The D-STATCOM has been utilized mainly for regulation of voltage, correction of power factor and elimination of current harmonics. It provides a continuous voltage regulation. In this paper, the D-STATCOM is used to regulate the voltage at the point of connection. The control is based on sinusoidal PWM and only requires the measurement of the rms voltage at the load point [3]. The DSTATCOM has the capability of generating continuous variable inductive or capacitive shunt compensation at a level up to its maximum MVA rating [4].

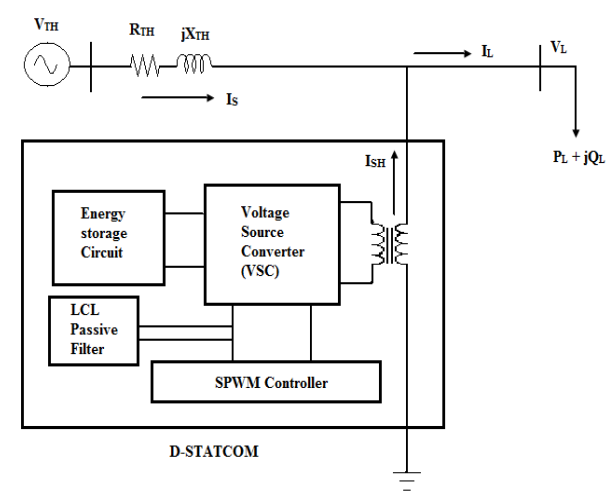

Fig 1: DSTATCOM schematic representation 


\section{FUZZY LOGIC CONTROLLER}

The Fuzzy Logic Controller (FLC) is used as the controller. The Fuzzy Logic tool was introduced by Lotfi Zadeh. It is a mathematical tool for dealing with uncertainty. In fuzzy logic, basic control is determined by a set of linguistic rules which are the user defined system. Fuzzy Logic Controller can be divided into main functional blocks namely knowledge base; fuzzification; Inference mechanism and defuzzification Rule Base.

\section{A. Error Calculation:}

The error is calculated as the difference between supply voltage data and the reference voltage data and the error rate is the rate of change of error.

\section{B. Fuzzification:}

Fuzzification is a process; where the crisp quantities are converted to fuzzy. Fuzzification process involves assigning membership values for the given crisp quantities. This unit transforms the non-fuzzy input variable measurements into the linguistic variable that is a clearly defined boundary. In this simulation study, the error and error rate are defined by linguistic variables such as negative $\operatorname{big}(\mathrm{NB})$, negative medium(NM), negative small(NM), zero(ZE), positive small(PS), positive medium(PM) and positive big( $\mathrm{PB})$.

C. Decision Making:

Fuzzy process is implemented with Mamdani method. Mamdani inference method easily obtains the relationship between its inputs and output. The decision rules are represented is given in table 1

TABLE I FUZZY DECISION RULES

\begin{tabular}{|c|c|c|c|c|c|c|c|}
\hline $\mathrm{e} / \Delta \mathrm{e}$ & $\mathrm{NB}$ & $\mathrm{NM}$ & $\mathrm{NS}$ & ZE & PS & PM & PB \\
\hline NB & NB & NB & NB & NB & NM & NS & ZE \\
\hline NM & NB & NB & NB & NM & NS & ZE & PS \\
\hline NS & NB & NB & NM & NS & ZE & PS & PM \\
\hline ZE & NB & NM & NS & ZE & PS & PM & PB \\
\hline PS & NM & NS & ZE & PS & PM & PB & PB \\
\hline PM & NS & ZE & PS & PM & PB & PB & PB \\
\hline PB & ZE & PS & PM & PB & PB & PB & PB \\
\hline
\end{tabular}

D. Defuzzification:

It is the process of converting the controller outputs to control signals. Defuzzification means the fuzzy to crisp conversions by using Centroid method. [5]

\section{METHODOLOGY}

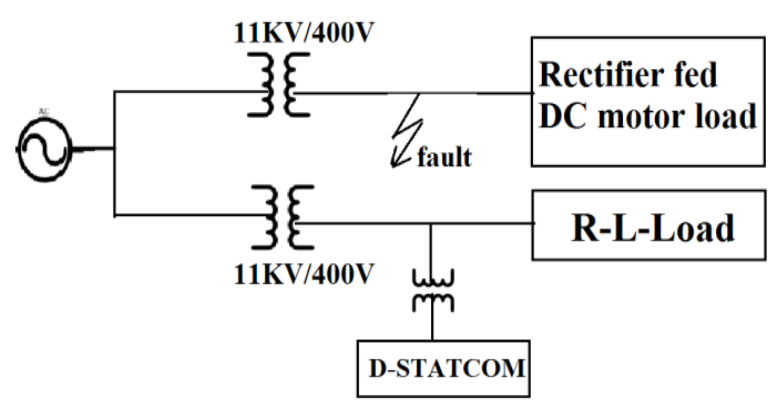

Fig 2: single line diagram
The entire system can be represented in single line diagram as in figure 2

The system parameters are given as below

\begin{tabular}{|l|l|}
\hline Source voltage & $11 \mathrm{KV}$ \\
\hline $\begin{array}{l}\text { Distribution transformer } \\
\text { rating }\end{array}$ & $11 \mathrm{KV} / 400 \mathrm{~V}, 63 \mathrm{KVA}$ \\
\hline DC Motor rating & $50 \mathrm{H} . \mathrm{P}$ \\
\hline RL Load & $5 \mathrm{KVA}(0.8 \mathrm{p.f} \mathrm{lag})$ \\
\hline DC Link voltage & $200 \mathrm{~V}$ \\
\hline transformer ratio & $1: 2$ \\
\hline Filter Inductance & $6 \mathrm{mH}$ \\
\hline Filter Capacitance & $800 \mu \mathrm{F}$ \\
\hline Carrier frequency & $2000 \mathrm{HZ}$ \\
\hline Armature voltage & $450 \mathrm{~V}$ \\
\hline Excitation Voltage & $200 \mathrm{~V}$ \\
\hline
\end{tabular}

The system be implemented by using MATLAB Simulink in figure 3 and simulink model of DC motor in figure 4

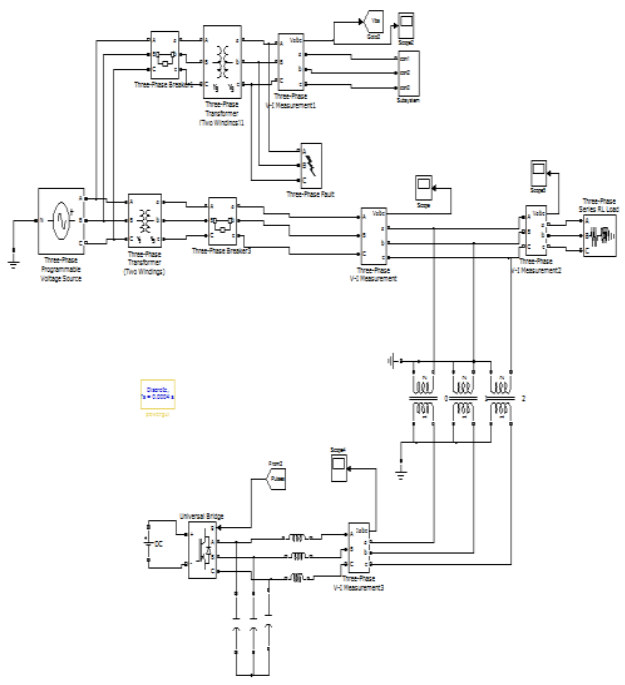

Fig 3 : simulation model for proposed method

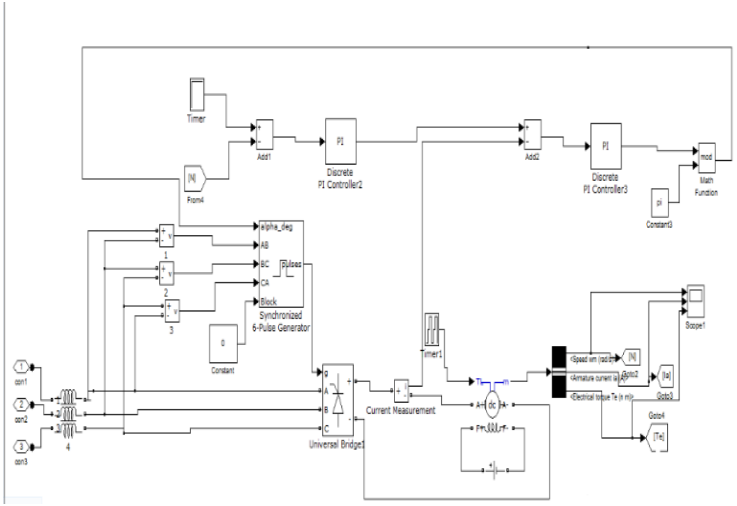

Fig 4 :simulink model of motor

PARK'S TRANSFORMATION:

DSTATCOM control employs a-b-c to d-q-0 transformation. During the abnormal conditions, the voltage change. After comparing d-voltage and q-voltage with the desired voltage error, $\mathrm{d}$ and $\mathrm{q}$ are generated. This 
error component is converted into

a-b-c component using d-q-0 to a-b-c transformation.

\section{CONTROL CIRCUIT:}

The reference voltage and the load voltage is converted from three phase quantity to two phase quantity using parks transformation and from the difference error is calculated. Error rate and error is given as input to fuzzy controller to get the actuating signal. The actuating signal is converted from two phase to three phase quantity as a sinusoidal waveform. Sinusoidal waveform is compared with triangular carrier signal. When the control signal is greater than the carrier signal, three switches of the six are turned on and the counter switches are turned off with the triggering pulses. The fuzzy control circuit is implemented in figure 5 with error membership function in figure 6 ,error rate membership function in figure 7 and output membership function in figure 8

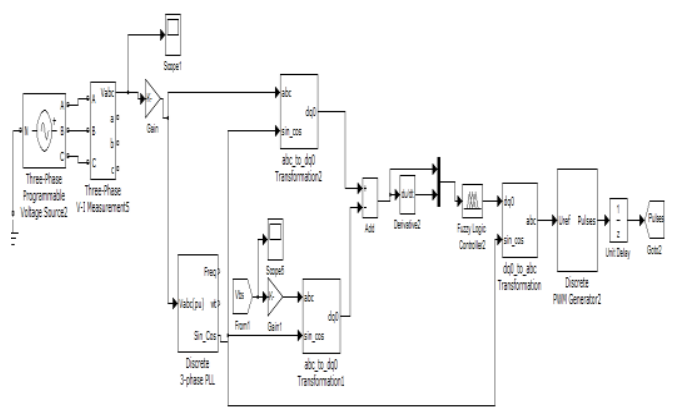

Fig 5: simulink control circuit for fuzzy control

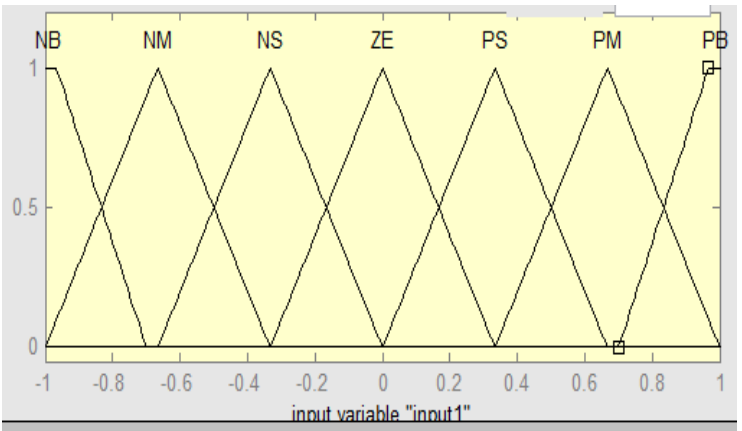

Fig 6: input membership function for error

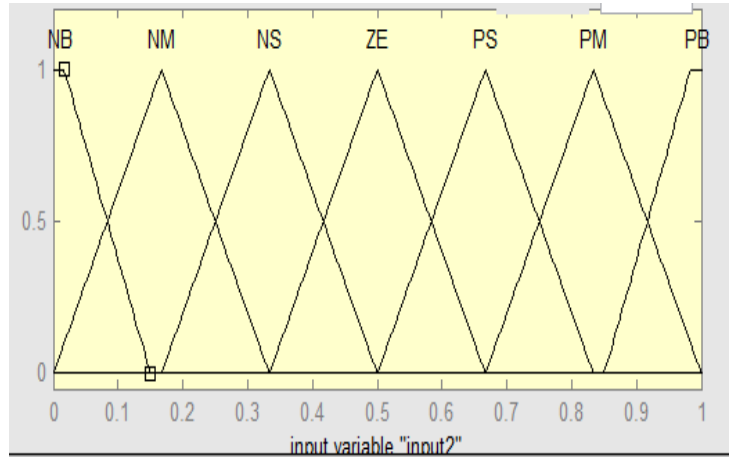

Fig 7: input membership function for error rate

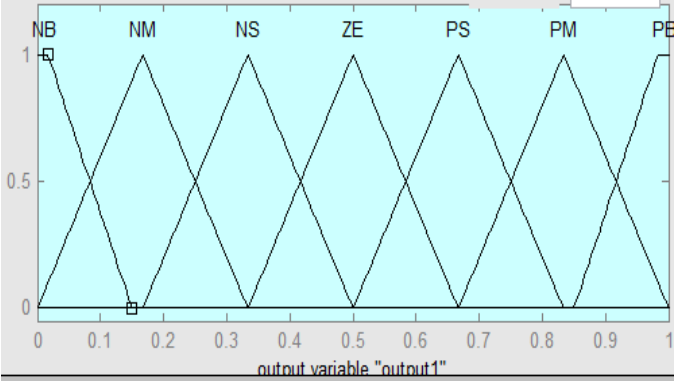

Fig 8:output membership function

\section{LG FAULT:}

When LG fault occurs on feeder which feeds DC motor load the voltage sag occurs on the RL load. As RL load is connected to same finite source. voltage restoration is done by using DSTATCOM Current; DSTATCOM injects three phase current. Thus the voltage is restored by using DSTATCOM.

The voltage sag occurs in voltage waveform from 0.1 to 0.2 duration represented in figure 9.the DSTATCOM current is shown in figure 10 and the restored voltage be given in figure 11 .

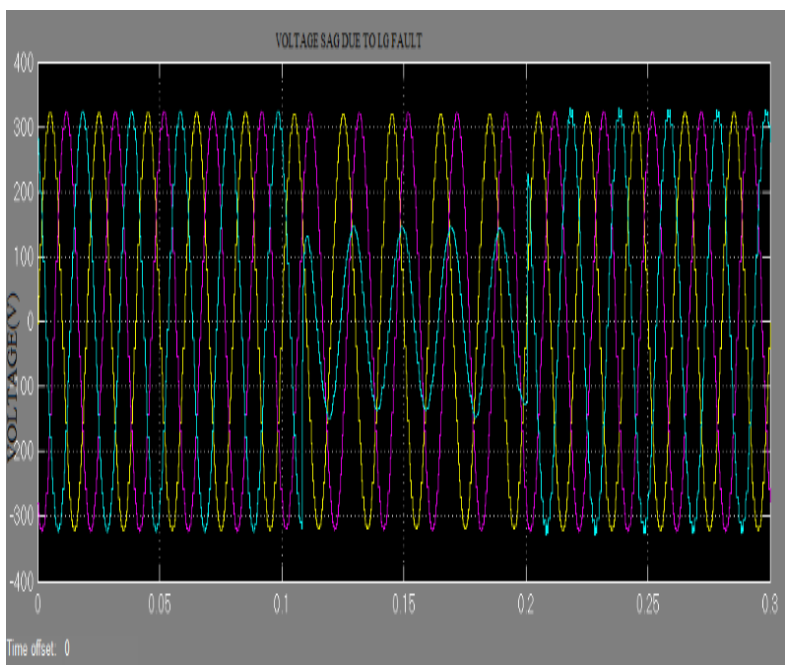

Fig 9: voltage sag due to LG fault

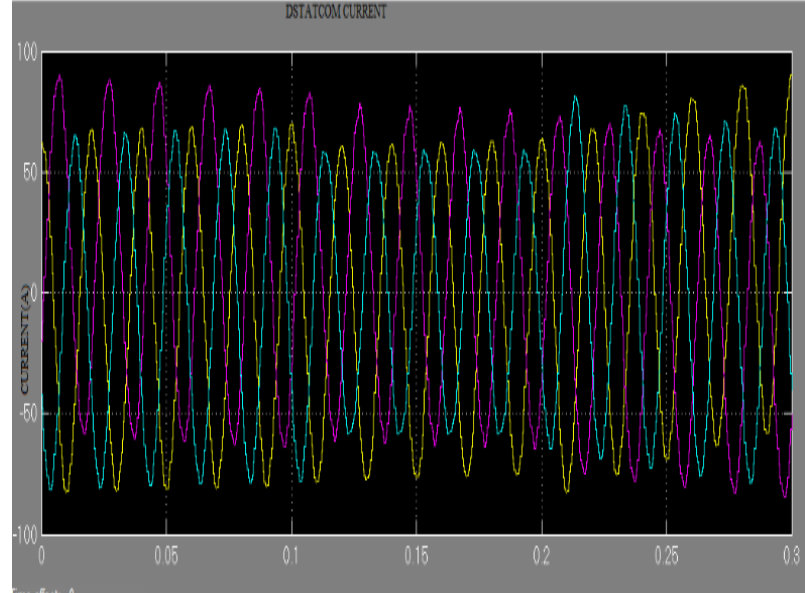

Fig: 10DSTATCOM CURRENT 


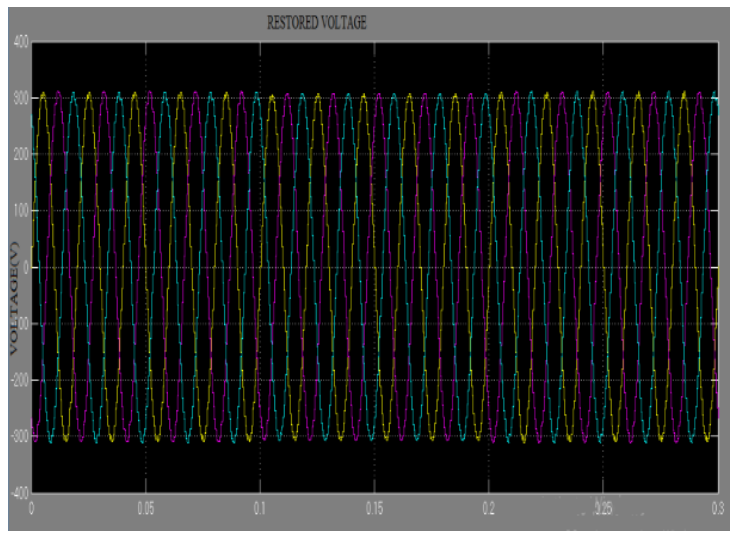

Fig 11: Restored voltage

\section{LL FAULT:}

Whenever LL fault occurs on the DC Motor load feeder the voltage on the RL load affects. As the RL load is connected to same finite source. DSTATCOM induces three phase current for the voltage restoration.

The System is simulated with fuzzy logic controller and LL fault on the DC motor load which causes sag from the duration of 0.1 to 0.2 on the RL load is represented in figure 12.the induced DSTATCOM current is in figure 13 and restored voltage in figure 14 .

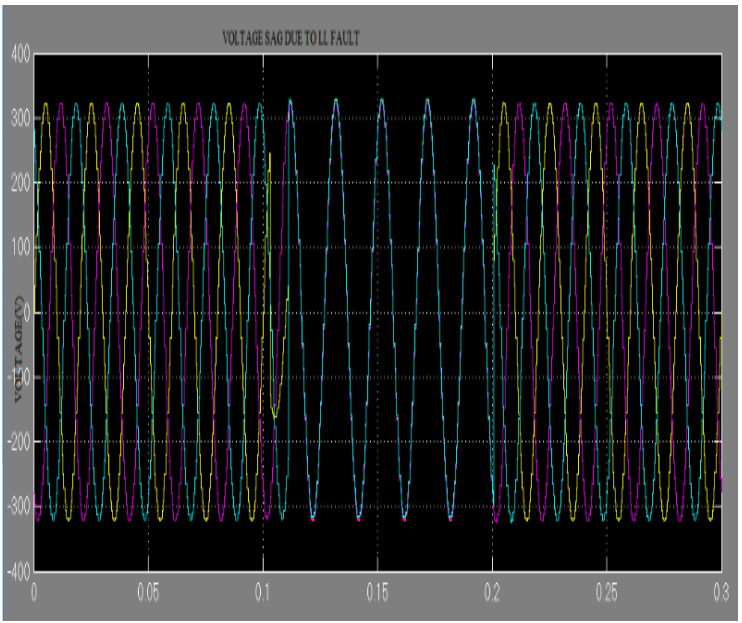

Fig 12: voltage sag due to LL fault

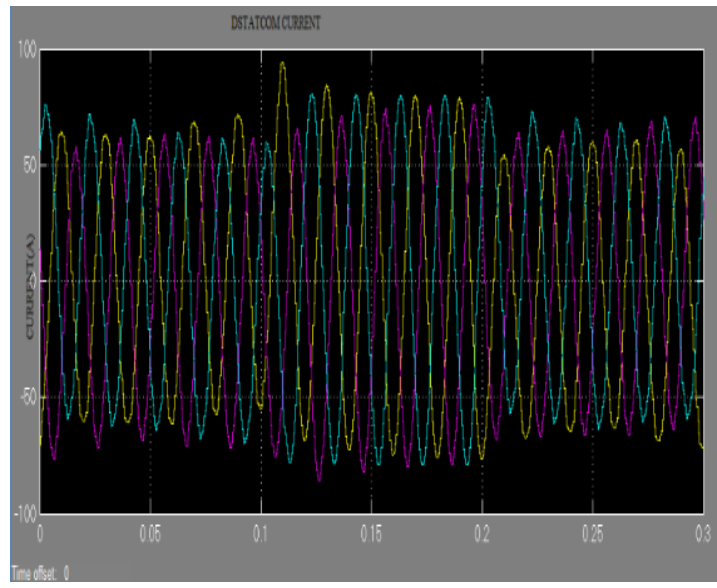

Fig 13: DSTATCOM current

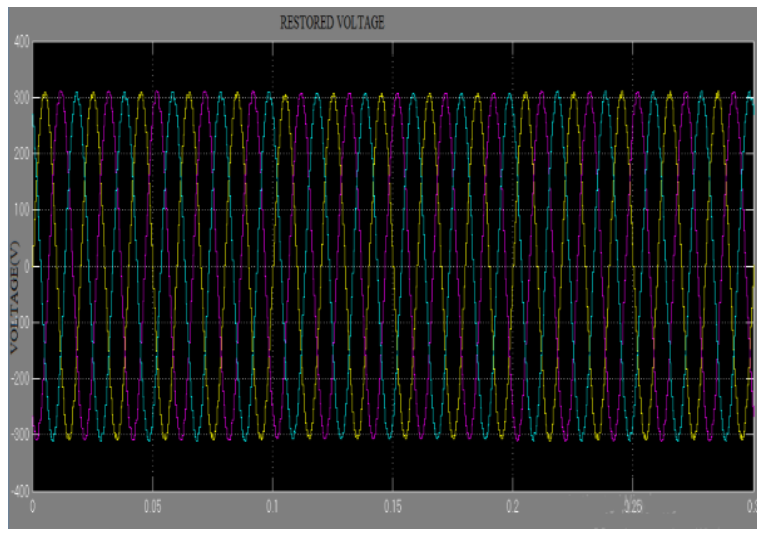

Fig 14: restored voltage

\section{IV.CONCLUSION}

DSTATCOM with Fuzzy controller is applied to compensate voltage sag. Fuzzy controller is better than conventional PI controller. The fuzzy logic controller provides much better result, faster and smoother response than the conventional PI controller. Thus Fuzzy is robust nature. The D-STATCOM corrects all the voltage magnitudes, phase deviations and harmonics at the desired load point. The simulation results clearly indicates that DSTATCOM provide excellent voltage compensation capability.

\section{REFERENCES}

[1]. Design and Simulation Studies of D-STATCOM for Mitigating Voltage Sag Problem by Using Fuzzy Inference System, and Proportional Integral based on controlled application Hamza Jaber Mohamed, Mohamed Ali Abdulsalam, Fathi A Mansur, Miftahul Anwar ,Muhammad Nizam .

[2]. Solution of Power Quality Problems using DSTATCOM by Ramesh C. Kumhar, Nitin Sandhya, Prakash Sundaram,Pankaj Kumar, Raju Swami

[3]. Mitigation of power quality problems by using DSTATCOM by Shaik Khaja Gareeb Nawaz, Shaik Hameed

[4]. VSC Based DSTATCOM \& Pulse-width modulation for Power Quality Improvement by Rodda Shobha Rani, B. Jyothi

[5]. Fuzzy Logic Control of D-Statcom for Power Quality Improvement by D. Prasad, T. Sandeep Kumar, Dr.B.Venkata Prasanth, Dr.K.S.G.Sankar

\section{BIOGRAPHIES}

T.Divya Sree, PG Student in V.R.Siddhartha engineering Vijayawada. Areas of interest are Power System, Power Electronics.

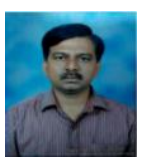

M. Devi Shankar working as a Associate Professor. He has 14 years of teaching experience. He has published many papers in national and international conferences and journals. He is currently working in V.R.Siddhartha College. His areas of interest are Power Electronics, Industrial drives, Artificial Intelligence. 